\title{
La educación sexual debe comenzar en el hogar y continuar en la escuela
}

\author{
Publicado en Revista Chilena de Pediatría 1931; 384-387
}

\author{
Autores: W. COUTTS Y G. MORALES BELTRAMI. \\ Comentario: LUISA SCHONHAUT B. ${ }^{1}$ y TERESA MILLÁN K. ${ }^{2}$ \\ 1. Departamento de Pediatría, Facultad de Medicina Clínica Alemana - Universidad del Desarrollo. \\ 2. Departamento de Pediatría Campus Occidente, Universidad de Chile.
}

Key words: Pediatric history, sexual education, antivenereal campaign.

Palabras clave: Educación sexual, lucha antivenérea, historia pediatría.

"Nuestro cuerpo es sensible y sexuado desde la cabeza a los pies y la construcción de la sexualidad nace cuando nos es dada la vida y en un proceso de constante comunicación y enriquecimiento individual y social”

(TMK 2010)

\section{Introducción: contexto histórico}

La sexualidad es una esfera que abarca todas las dimensiones de la persona biológica, psicológica y socialmente, es por tanto, una parte inherente e integral del ser humano desde que nace y a lo largo de toda su vida.

"La sexualidad es concebida como una apetencia natural al ser humano tal como lo son comer, beber, trabajar, dormir, divertirse. Sin embargo, algo hace que no se pueda hablar de ella abiertamente como lo hacemos del comer o del trabajar. Ninguna surge tan rodeada de misterios, tan necesitada de privacidad y ocultamiento, tan ligada intrínsicamente a exigencias éticas, como el sexo."(A. Roa 2007) ${ }^{1}$. En nuestra tradición occidental, la sexualidad ha sido un tema manejado en forma de tabú, siendo el país testigo de un choque cultural, entre un pueblo indígena que vivía la sexualidad en forma libre y natural versus un nación española clerical y represiva.

En este escenario de pecados y tabúes, los pediatras y ginecólogos no podían mantener una actitud pasiva, debido a que las enfermedades de transmisión sexual daban cuenta de un elevado porcentaje de jóvenes infectados y eran una de las principales causas de mortalidad general e infantil. Si bien este era un problema de salud pública a nivel universal, la prevalencia en nuestro país era aún mayor ${ }^{2}$. Estadísticas de la época refieren que durante la primera mitad del siglo XX un alto porcentaje de la población estaba contagiada de sífilis y gonorrea, 70\% de los infectados tenía entre 16 y 25 años y se calculaba que cerca del 3\% correspondía a niños y adolescentes ${ }^{3}$; la sífilis congénita era causante del 8,7\% de la mortalidad de niños menores de 1 año y la principal causa de mortintalidad ${ }^{4}$.

Correspondencia a:

Luisa Schonhaut B.

E-mail: Ischonhaut@alemana.cl 
Coutts publicaba el año 1931 las estadísticas de seguimiento de 358 "madres chilenas sifilíticas", entre las que se contabilizaron 1.339 embarazos, cuya evolución fue "abortos 557, nacidos muertos 123; fallecidos en la primera infancia 292 y vivos a la fecha del examen sólo 367"'. Por otro lado, en el análisis de los prematuros ingresados a la Casa Nacional del Niño, el año 1941, la lúes materna, aislada o asociada a otra causa de prematurez, daba cuenta de "la mortalidad más alta con respecto a un factor etiológico de prematurez determinado" 6 .

Del mismo modo, el Dr. Salvador Allende consideraba la campaña antivenérea como parte de la "lucha contra la delincuencia", otro de los importantes problemas que aquejaba nuestro joven país. "Existen individuos que se caracterizan por su psiquismo negativo, que son capaces de cometer los más crueles delitos, $y$ son aquellos que debido a alteraciones o atrofias del aparato genital, originadas por enfermedades venéreas, cambian su carácter y su personalidad, y de individuos honrados y laboriosos pueden convertirse en seres peligrosos para la armonía social." Para él, la alta prevalencia de enfermedades de transmisión sexual en la población joven era explicable por factores fisiológicos y sociales, “...debido a una pubertad temprana, que sería el factor fisiológico, y al desconocimiento absoluto en que vive la mayoría de ellos respecto de los más elementales preceptos de medicina preventiva y su nula educación sexual. Este sería el factor social." (Allende, Tesis para optar al título de Médico, 1933) ${ }^{3}$

En una época en que la biología del desarrollo puberal y la sexualidad aún eran desconocidos, era necesario enfrentar enérgicamente el factor social, esa "onda de sexualismo que se ha desencadenado sobre el mundo civilizado". (Coutts 1933) ${ }^{7}$. La lucha antivenérea se convirtió en uno de los ejes principales de las Políticas Sanitarias de la primera mitad del siglo $\mathrm{XX}$, lo que implicó abrir el tema de sexualidad y enfermedades relacionadas y liberarlos de la estigmatización religiosa, para poder realizar una prevención efectiva ${ }^{8}$.

En el año 1927 el Gobierno organizó la “Lucha Antivenérea” como un Programa a nivel nacional, siguiendo los modelos que se habían probado en el viejo mundo. Se implementaron estrategias curativas o directas, y medidas indirectas que incluían una intensa campaña de propaganda y la distribución de "pomadas profilácticas" 3 . Los mensajes de auto cuidado y sexualidad responsable, como claves para tener hijos sanos, eran trasmitidos incluso en las cartillas de Puericultura, repartidas a todas las madres del país, en ellas se recomendaba "Si Ud. es sifilítico, tuberculoso, alcohólico o toxicómano (cocaína, morfina, etc. etc.), no engendre un hijo antes de haberse puesto en curación. La sífilis, el alcoholismo, la toxicomanía, degeneran la raza y producen en los hijos atraso intelectual, instintos perversos, locuras, epilepsia, etc"'.

En este contexto fue planteada por primera vez en nuestro país la educación sexual. Coutts proponía "enseñar desde temprano a los niños en las escuelas, higiene sexual y la manera de prevenir los males venéreos; educar a los padres y convencer a los moralistas del error. Sólo en esta forma haremos un bien frente a males que no tienen otra solución"7.

Para que la estrategia antivenérea fuese efectiva, era necesario reclutar a los distintos actores sociales, incluyendo los pediatras; seguramente fue ese el motivo que llevó a publicar el artículo "La educación sexual debe comenzar en el hogar y continuar en la escuela" en la revista chilena de pediatría el año 1931; en él, Waldemar Coutts, entonces jefe técnico de la Sección de Higiene Social de la Dirección de Sanidad y Guillermo Morales Beltrami, subjefe técnico de la misma división, nos ilustran acerca del debate respecto a la educación sexual que se encendió hace más de 75 años, y se mantiene, con diferentes matices hasta nuestros días.

\section{Artículo}

Hace ya muchos años que se habla de la necesidad de una educación sexual entre los niños y numerosos son, a la fecha, los países que han instituido esta práctica en sus escuelas a la vez que luchado por ilustrar a los padres respecto a los deberes frente a su descendencia. 
La cosecha ha sido fructífera, pero no lo suficiente para justificar los esfuerzos y dinero gastados con estos fines. La razón de ello reside principalmente, a nuestro juicio, en el hecho que no todos los padres tienen las mismas creencias; mientras unos comprenden la realidad de los hechos que la ciencia se esfuerza por demostrarles, otros no quieren oír la voz compasiva de sus semejantes y se atrincheran detrás de preceptos doctrinarios.

El niño, como todos los animales, tiene sus instintos y tendencias propios; pero no conocemos su alcance hasta que los interpretamos en términos sociales. Con estos fines debemos estar capacitados para situarlos en un estado social embrionario y considerarlos como supervivientes de actividades primitivas vinculadas a infinitos antepasados.

Si se suprime o cohíbe el desarrollo normal de la personalidad del niño, si se traba la libre expresión o satisfacción de sus deseos, se despierta en él, con frecuencia, una reacción antagónica compensadora que lo transforma en un rebelde contra todo precepto moral durante el resto de su vida. Es esta, a nuestro juicio, la razón porque a menudo los hábitos perniciosos adquiridos durante los primeros cinco años se arraigan profundamente en los niños.

Muchos autores han protestado contra los prejuicios y el olvido de la niñez propia al suponer que la infancia es asexual o presexual. Las inmensas alteraciones que se registran en la pubertad no nacen de la nada; antes de llegar a este estado existe, sin lugar a dudas, un desarrollo gradual de las que más tarde serán características fundamentales de uno y otro sexo.

La importancia de las glándulas sexuales en la determinación de aquellos cambios somáticos que constituyen el dimorfismo sexual, esta ya fuera del campo de la especulación científica. Sin embargo, aun no conocemos la manera precisa en que estos profundos cambios se efectúan; pero no nos asiste dudas que se inician desde los primeros instantes de la fecundación del ovulo por el espermatozoide y se acentúan ya en las primeras etapas de la vida intrauterina.

La teoría de las secreciones internas coloca a las glándulas sexuales al centro de las causas de la pubertad. A la castración prepuberal o a la hipoplasia de las gónadas, la serie de fenómenos que constituyen el complejo puberal o no se presentan o lo hacen tardíamente y deformados, aún cuando los procesos individuales de desarrollo se manifiesten en forma precisa. En consecuencia, tenemos que considerar el creciente radio de acción de las glándulas sexuales como el punto principal al cual se agregan progresivamente y en forma secundaria todas las manifestaciones y actividades de la pubertad.

Durante el curso de la vida del niño, el sistema endocrino no sufre un desarrollo uniforme. Sus distintas glándulas se desenvuelven, tanto en estructura como en tamaño, relativamente independientes unas de otras. Esta desigualdad de su desarrollo en el individuo normal y sus diversas actividades es lo que determina la hegemonía de las características de tal o cual de ellas; sin embargo, esta circunstancia no perturba su cooperación a la obra común de desarrollo. Pero en muchos niños, ya por herencia patológica (lúes, alcoholismo, etc.), por deficiente alimentación (avitaminosis materna o parvularia), influencias climáticas, etc., esta armonía se destruye y algunas glándulas endocrinas sufren las consecuencias más intensamente que otras, dando origen a cuadros de hipogenitalismo o hipergenitalismo con precocidad psico-sexual.

Entre estos cuadros nosológicos extremos no hay abismos sino una serie de alteraciones intermediarias íntimamente relacionadas con un mayor o menor funcionamiento de las glándulas endocrinas y que no podemos reconocer sin un estudio detenido del caso. Esta es la razón, a nuestro juicio, por la cual antes de iniciar la educación sexual es necesario conocer muy bien el terreno donde se va a arrojar la semilla.

La educación sexual debe empezar en el hogar. No hay duda, pero todos los padres no están igualmente capacitados para hacerla ni todos los niños aptos para recibirla en una misma época de la vida. Es esta la razón por la cual estimamos que es el médico que ha vivido más próximo del hogar quien debe señalar a los padres cuando debe iniciarse esta educación.

Él, mejor que nadie, conoce a los pequeños; 
los ha atendido desde sus primeras enfermedades; esta interiorizado acerca de los males que aquejan o han aquejado a sus progenitores, $\mathrm{y}$ este espíritu observador severo y disciplinado podrá juzgar cuando ha llegado la época de iniciar al pequeño, en forma lenta y gradual, sobre los misterios de la vida.

Difícil es para el médico encargarse personalmente de la educación de estos niños; sin embargo, expresándoles a los padres la necesidad y conveniencia de empezar esta clase de educación, habrá hecho un gran beneficio para la vida futura de sus pacientes.

La educación sexual en el hogar está al alcance de aquellos padres que tienen cierto grado de cultura, y aún en muchos de estos casos nos harán la siguiente pregunta: ¿como se lo digo a mis hijos? A ella respondemos: solicitando folletos editados por las Oficinas de Higiene Social de los Servicios de Sanidad.

En ellos encontraran los padres todo lo que deseen saber y la forma como pueden iniciar, de manera sencilla, la educación sexual de sus hijos.

Estos primeros pasos dados mano a mano con sus padres, despertaran la confianza entre ellos y sus hijos y estos podrán, cuando se encuentren frente a problemas de difícil solución, siempre contar con la ayuda generosa y experimentada de sus progenitores.

Frente a la falange de padres que desean educar a sus hijos y que pueden hacerlo, nos encontramos con una masa inmensa de niños, cuyos padres pregonan un ascetismo que no cumplieron o que, por su escaso nivel cultural, no están capacitados para enseñar lo que ellos mismos ignoran.

Corresponde al Estado, en estos casos, proporcionar a esos niños los medios de llegar a comprender la razón de su existencia y el origen de su vida. En la escuela, sea cual fuere su naturaleza, debe hacerse educación sexual. Cada médico escolar, asesorado por un psicólogo, debe estudiar detenidamente a los colegiales y analizar sus condiciones físicas para indicar cuándo deben asistir a las clases donde se traten estos temas.

Una vez controlados y catalogados, deben ser llevados por grupos los niños de diversos colegios a recibir enseñanza colectiva en loca- les donde se harán clases sencillas y se pasarán películas ilustrativas, empezando por explicarles la polinización de las plantas y, a medida que llegan a mayor edad, se les enseñarán los fenómenos inherentes a la fecundación para concluir, en los últimos cursos, con la esquematización de las enfermedades de los órganos de la reproducción y la manera de evitarlas.

A estas conferencias debe procurarse que asistan los padres conjuntamente con sus hijos; ello ilustrará a los ignorantes y afianzara los lazos de unión espiritual que deben existir entre padres e hijos.

$\mathrm{Si}$, como ya hemos manifestado, no debe concederse privilegios a determinadas escuelas para prescindir de enseñar la verdad; estimarnos que tampoco debe hacerse diferencia entre la enseñanza de niños o niñas. Ellas también deben conocer el significado de los fenómenos naturales.

Que una educación sexual amplia como la que proponemos va a despertar precozmente a los niños en la sexualidad, exclamaran los moralistas. Profundo error! Nadie escapa a la tiranía de sus propias células; es inútil cambiar el mundo celular íntimo; el ascetismo, la abstinencia que pregonan algunos es sólo posible entre Santos.

Si el niño se aventura a la relación sexual, lo hace impulsado por una corriente ancestral peculiar de su especie y es preferible que entre a cumplir con la función de la cópula a sabiendas de lo que puede acontecerle, ya que en esta forma conocerá las precauciones que debe tomar y, si es enfermedad, sabrá a quien debe recurrir en su hora de desgracia.

La educación sexual amplia tiene además, a nuestro juicio la ventaja de despejar desde temprano una incógnita que preocupa a los niños. Cuando son púberes o post-púberes, su ignorancia sobre estos asuntos les da sobrado tema para conjeturas y especulaciones que no hacen otra cosa que incitarlos a conocer los misterios y encantos que pregonan los iniciados. Sabiendo la verdad, el encanto de lo extraño, de lo nuevo, desaparece y otras múltiples preocupaciones concluyen por adormecer un instinto que se desarrolla hacia un fin normal.

Que llega una época en que tendrán que cumplir con la función natural antes que los 
lazos sagrados del matrimonio los una a una mujer o a un hombre, es lógico y razonable. Nuestra actual manera de vivir, el rodaje de la vida contemporánea es tal, que nunca nos permitirá que hagamos de nuestros hijos abstinentes. Día a día las estadísticas sobre contagio venéreo revelan que los sexos fisiológicamente aptos para la procreación se unen con mayor precocidad con el fin de cumplir una función natural. Y me atrevería a preguntar de aquellos que escuchan la confesión de almas que buscan perdón, si no es efectivo que en nuestro siglo ha aumentado inmensamente el número de pecados sexuales tanto entre niños de las altas clases sociales como en las bajas.

Estos hechos son más elocuentes que lo que predican los necios y escuchan los pequeños de espíritu, acerca de la aberración que significaría la educación sexual de nuestra juventud. Es necesario prevenir para curar; si no enseñamos a quienes mañana ocuparán nuestros lugares en el drama universal, no atino a comprender que respuesta daremos cuando el Supremo nos llame a rendir cuenta de nuestros actos y llegue a preguntarnos: ¿qué hiciste por tus hijos?

En vista de los hechos que acabamos de señalar estimamos que:

1. El estado debe propender, por todos los medios a su alcance, a la educación de los padres en el sentido que los fenómenos naturales no deben ser señalados a los hijos como misteriosos o faltos de moralidad.

2. La educación sexual debe ser obligatoria en las escuelas de ambos sexos, sea cual fuese su naturaleza.

3. Debe iniciarse entre los niños escolares cuando el médico del establecimiento y el psicólogo asesor lo estimen oportuno.

\section{Comentario: la educación sexual en nuestros días}

El desarrollo de programas de educación sexual en nuestro país tuvo su comienzo en los años 30, en el marco de una campaña Nacional de Lucha Antivenérea. "La educación sanitaria constituye una preocupación constante de la sección. A fin de mantener el contacto con el cuerpo médico, se ha establecido un sistema de encuestas periódicas. En una se solicitó la opinión de los venereólogos de los puntos donde hay policlínicas, acerca de si habían o no comprobado disminución en la frecuencia de los nuevos casos de sífilis. En Santiago, de 20 interrogados respondieron 11, 9 de ellos afirmativamente. Otro cuestionario dirigido a los pediatras con respecto a si habían observado disminución en el número de heredosifilíticos, recibió 9 contestaciones entre 20 interrogados, contestando 6 que habían observado disminución en los heredosifilíticos, y 7 en el número de los niños que se presentan con manifestaciones tardías de sífilis" ${ }^{\prime 10}$. Si bien estos resultados podrían haber sido interpretados como una señal favorable, las medidas profilácticas no fueron suficientes y la prevalencia de gonorrea y sífilis continuó en aumento hasta la llegada de la penicilina en los 40, con lo que se logró finalmente su control. No sería hasta los años 80, en que, con la aparición de un nuevo enemigo venéreo, el VIH/SIDA, las estrategias de educación sexual cobraron un nuevo impulso ${ }^{8}$.

Si bien habría consenso sobre la importancia de la educación sexual y que esta "debe empezar en el hogar y continuar en la escue$l a "$, considerando las múltiples aristas tanto religiosas como valóricas y culturales que matizan este tema, a lo largo de la historia ha habido controversia respecto a quiénes son los que deberían enseñarla a los niños y adolescentes. Mientras los jóvenes adjudican la responsabilidad a sus padres, a quienes visualizan como sus aliados naturales y referentes principales $^{11}$, los adultos piensan que dicho compromiso debe ser compartido con la institución educacional ${ }^{12}$.

Las barreras para la implementación de programas de educación sexual antaño eran múltiples. El gran problema era que los padres en un alto porcentaje eran analfabetos o carecían de la preparación necesaria para enfrentar la temática con sus hijos; "La educación sexual en el hogar está al alcance de aquellos padres que tienen cierto grado de cultura, y aún en muchos de estos casos nos harán la siguiente pregunta: ¿cómo se lo digo a mis hijos?”. (Coutts y Morales Beltrami 1931). Por otro lado, era difícil continuar la educación en la escuela, dado que las tasas de escolaridad eran muy ba- 
jas. Por este motivo, el gobierno a través de la Dirección General de Sanidad generó planes educativos para las escuelas, cursos para los padres y publicó folletos educativos que abordaban distintas temáticas.

En aquellos años los pediatras, ya sea como médicos de cabecera o como médicos escolares, tenían un rol en la determinación del momento propicio para comenzar la educación de los jóvenes, con su "espíritu observador severo y disciplinado podrá juzgar cuando ha llegado la época de iniciar al pequeño, en forma lenta y gradual, sobre los misterios de la vida." (Coutts y Morales Beltrami 1931) Probablemente el presente artículo fue publicado con el fin de motivar a los pediatras y reforzar su responsabilidad en la tarea educar para prevenir las enfermedades de contagio venéreo.A lo largo de los años, esta realidad ha ido cambiando, gracias al aumento de la cobertura de educación de las familias y, a la facilidad de acceso a la información, que otorgan los medios tecnológicos informáticos de comunicación. Quizás por ello, en nuestros días los pediatras han perdido protagonismo, de modo que cuando de responsabilidades se habla, los profesionales de salud de Atención Primaria aparecen mencionados en último lugar, siendo las matronas señaladas en mayor proporción ${ }^{13,14}$.

En una investigación reciente se evidenció que $77,6 \%$ de las madres y $68 \%$ de los padres de adolescentes de la región Metropolitana reconocía la inquietud en relación a la sexualidad de sus hijos/as. La orientación sobre cómo prevenir enfermedades de transmisión sexual, VIH/Sida y el embarazo, fueron las necesidades reconocidas como las más importantes, sin diferencias por sexo de los hijos. En la mayoría de los hogares con adolescentes se conversaba sobre temas de sexualidad ${ }^{14}$.

En cuanto a la educación de salud sexual y reproductiva en los colegios, estudios realizados en distintas épocas han evidenciado la falta de cobertura de dichos programas ${ }^{15}$. En la reforma educacional impartida hace una década se incluyeron contenidos de salud sexual y reproductiva en el currículo de la enseñanza media, como parte del curso de ciencias naturales. Pero considerando que a los 12 ó 13 años un porcentaje de adolescentes ya ha comenzado su vida sexual activa, muchas veces producto de situaciones involuntarias, recientemente se determinó que la obligatoriedad de incorporar la sexualidad en el currículo a partir de los primeros años de educación.

Al repasar la historia, los contenidos del artículo de Wlademar Coutts y Guillermo Morales Beltrami aún se encuentran vigentes. Tanto ayer como hoy los padres y madres se reconocen como los principales protagonistas para educar sobre sexualidad, sin embargo, al momento de desear hacerlo se encuentran poco capacitados y sienten que necesitan ayuda para ello. ¿Qué los frena o cuáles son las barreras que enfrentan para poder asumir esta tarea?, ¿Por qué les es difícil sobre todo cuando se trata de diferente género del hijo?, ¿Qué pasa cuando se dialoga sobre la intimidad de su sexualidad con los hijos e hijas adolescentes?

¿Cómo se lo digo a mis hijos? Quizás es la pregunta que resume las inquietudes planteadas por Coutts hace casi un siglo, continúan preocupando a los padres y a los profesionales de salud y educación; quizás para responderlas debamos escuchar más a nuestros adolescentes, ya que son ellos quienes nos pueden dar la clave para encontrar las respuestas. Sin duda, para favorecer el desarrollo pleno y armónico de nuestros hijos e hijas, debemos contribuir a la tarea de asegurar una educación integral, que incluya la sexualidad, mirada desde sus distintas aristas, tanto biológicas, pero también valóricas y morales.

\section{Referencias}

1.- Roa A: 2007 [citado 2011-08-21]. Disponible en: https:// www.u-cursos.cl/medicina/2007/1/MFAHEM22/1/ material.../2675.

2.- Allende S: La realidad médico-social chilena. Ministerio de Salubridad. Santiago, 1939. . [citado 2011-08-21]. Disponible en: http://www.memoriachilena.cl/archivos2/pdfs/MC0000543.pdf

3.- Allende S: Higiene mental y delincuencia: Memorias año 1933. Archivo Facultad de Medicina U. de Chile. Santiago. [citado 2011-08-21]. Disponible en: http:// www.salvador-allende.cl/Documentos/1939-49/MemoriaSAG.pdf 
4.- Schonhaut L: La Mortalidad Infantil en Chile estudiada por la Sociedad de las Naciones. Rev Chil Pediatr 2007; 78 (2): 202-10

5.- Coutts W: Las Enfermedades Venéreas y el Matrimonio. $3^{\circ}$ edición 1930. [citado 2011-08-21]. Disponible en: http://www.memoriachilena.cl/archivos2/pdfs/ MC0016113.pdf

6.- Schonhaut L, Pérez M: Estudio de 258 Prematuros en el Primer Año de Vida. Rev Chil Pediatr 2010; 81 (3): 253-60.

7.- Coutts W: citado en Allende S: Higiene mental y delincuencia: Memorias año 1933. Archivo Facultad de Medicina U. de Chile. Santiago. [citado 2011-08-21]. Disponible en: http://www.salvador-allende.cl/Documentos/1939-49/MemoriaSAG.pdf

8.- Labarca C: 'Todo lo que usted debe saber sobre las enfermedades venéreas': las primeras campañas de educación sexual estatales entre 1927 y 1938. In: Zárate, María Soledad. Por la salud del cuerpo: historia y políticas sanitarias en Chile. Santiago, Universidad Alberto Hurtado, 2008. p.81-129

9.- Schonhaut L: Cartilla de Puericultura de la Sociedad Chilena de Pediatría. Rev Chil Pediatr 2008;79 (1): 859.

10.- Coutts W, Morales Beltrami C: Bol. Dic. Gen. San. (jun.-dbre.) 1930. citado en Crónicas de la PAHO [citado 2011-08-21]. Disponible en: http://hist.library.paho. org/Spanish/BOL/v11n2p145.pdf

11.- Fernández L, Bustos L, González L, Palma D, Villagran $J$, Muñoz S: Creencias, actitudes y conocimientos en educación sexual. Rev Chil Pediatr 2000; 128: 574-83.

12.- Arancibia V: la educación en Chile: percepciones de la opinión pública y de expertos. Estudios Públicos, 54 (otoño 1994) [citado 2011-07-21]. Disponible en: http://curriculumygestion.blogcindario.com/ficheros/ laeducacionenchileestudiospublicos.pdf

13.- Pérez R, Cid M, Lepe, Carrasco C: Conocimientos, actitudes y comportamiento sexual en un grupo de adolescentes chilenos. Perinatol Reprod Hum 2004; 18 (4): 225-30.

14.- Luengo X, Millán T, Herreros J, Zepeda A, Henríquez ME, Montero A: Madres de adolescentes en Chile: ¿Qué saben y demandan respecto de la atención en salud sexual y reproductiva de los servicios de salud públicos que atienden adolescentes? Proyecto FONIS SA08I200499

15.- Millán T, Valenzuela S, Vargas A: N. Salud Reproductiva en adolescentes escolares: conocimientos actitudes y conductas en ambos sexos, en una comuna de Santiago de Chile. Rev Med Chile 1995; 123: 368-75. 